\title{
Branched-chain ignition in strained mixing layers
}

\author{
J D Mellado A L Sánchez J S Kim A Liñán
}

\begin{abstract}
The time-dependent evolution of the radical pool in an initially inert hydrogen-air counterflow mixing layer subject to variable strain is investigated analytically. Although the initial chemistry description contains three different chain carriers, namely, $\mathrm{H}, \mathrm{O}$ and $\mathrm{OH}$, it is shown that the ignition problem can be accurately described in terms of a single radical-pool variable that incorporates steady-state assumptions for the radicals $\mathrm{O}$ and $\mathrm{OH}$. Use of this non-standard procedure reduces the problem to the integration of a single conservation equation, whose solution depends on the existing Damköhler number $\Delta$, defined as the ratio of the diffusion time across the mixing layer to the characteristic branching time. Ignition takes place when $\Delta$ remains predominantly above a critical value of the order of unity. The exponentially growing radical pool, which extends across the mixing layer, can be described analytically by separation of variables in configurations with a slowly varying strain rate, providing a solution that is used to investigate the parametric dependences of the ignition time. Weakly strained solutions are studied separately by addressing the asymptotic limit of large Damköhler numbers. It is seen that the reaction zone then becomes a thin layer of relative thickness $\Delta^{-1 / 4}$ centred at the location where the branching rate is maximum. The analysis employs asymptotic expansions in decreasing powers of $\Delta$ for the shape and for the exponential growth rate of the radical pool. The accurate description of the solution necessitates computation of three terms in the asymptotic expansion for the growth rate, yielding predictions for the ignition time that remain accurate even for values of $\Delta$ of the order of unity.
\end{abstract}

\section{Introduction}

In many premixed and non-premixed combustion systems, combustion takes place in strained mixing layers. The solution that is found depends on the strain rate imposed by the outer flow field: large strain rates preclude combustion and yield quasi-frozen solutions, while weakly strained mixing layers favour chemical reaction, giving rise to the appearance of flames. In his work [1], Liñán took advantage of the large value of the activation energy typically found in combustion processes to calculate the S-shape curve of steady solutions for diffusion flames in strained, counterflow mixing layers, with a single irreversible Arrhenius reaction adopted for the chemistry description. In particular, he studied the ignition regime, corresponding to the quasifrozen lower branch of this S-shaped curve. He found that for strain rates below a critical value, the quasifrozen solution cannot exist, and a diffusion flame is formed. Critical conditions for ignition can now be routinely computed with codes that incorporate detailed 
descriptions for the transport and for the chemistry, and much information is now available for an increasing number of fuels.

On the other hand, only a limited number of papers have addressed the time-dependent combustion process leading to ignition in strained mixing layers. A related quantity, of particular interest in diesel combustion and also in supersonic propulsion applications, is the ignition time, or time required for the solution to evolve from a frozen state to a diffusion flame. All previous analytical studies [2-5] used a one-step Arrhenius model for the chemistry description. For instance, Niioka [2] calculated ignition times in a mixing layer subject to a constant strain rate, clearly showing how the existence of strain always retards ignition. The work of Linán and Williams [3] is of a more general nature, in that they considered both strain and pressure variations with time. After formulating the general problem, analytical solutions relevant for diesel ignition were developed. Other interesting contributions are those of Thévenin and Candel [4,5], who investigated the ignition distance in hydrogen-air shear flows. By following the downstream evolution of the interface, the problem of obtaining the ignition distance was reduced to the calculation of the ignition time in a mixing layer with time-dependent strain.

In this paper, we investigate chain-branching ignition in the hydrogen-air counterflow mixing layer subject to variable strain. Steady ignition solutions in this configuration were first investigated numerically by Darabiha and Candel [6]. They found that, for values of the strain rate above the critical ignition value, only trace amounts of radicals are present. The branch of ignited solutions emerging at the critical strain rate can be described as an imperfect bifurcation $[7,8]$, with transition from subcritical to supercritical behaviour being observed as the oxidizer temperature is increased [6-8]. In the present analysis, we extend the previous investigations by considering the unsteady process of chain-branching growth that takes place as the solution evolves from an inert state to eventually form a diffusion flame. The air temperature is assumed to be above the crossover temperature [9] corresponding to the second explosion limit of hydrogen-oxygen combustion, a limit in which the process reduces to a thermally frozen chain-branching explosion.

The paper is organized as follows. The problem is formulated in section 2 in terms of a variable Damköhler number, whose evolution in practical cases is investigated in section 3 . The chemistry description is reduced in section 4 , and a single variable is introduced as representative of the radical pool. The analytical solution corresponding to the case of slow strain-rate variations is presented in the following section. Weakly strained mixing layers are investigated next in section 6, with details of the required asymptotics being presented separately in an appendix. Finally, some conclusions are given in section 7.

\section{Formulation}

\subsection{Conservation equations}

The time-dependent conservation equations corresponding to a reactive counterflow mixing layer subject to variable strain are given below. For simplicity, the density, $\rho$, the specific heat at constant pressure, $c_{p}$, and transport properties are assumed to be constant, and a Fickian approximation is adopted for the transport of chemical species. In this thermo-diffusive approximation, the transverse velocity across the planar mixing layer reduces to the potential solution $v=-a x$, where $a(t)$ is the time-dependent strain rate and $x$ is the distance from the stagnation plane. In describing the solution, it is convenient to introduce a rescaled coordinate $\eta=x / \delta(t)$ which appropriately takes into account the changes in mixing-layer thickness, $\delta(t)$, that emerge due to the variable strain. This type of reduced formulation, which was first 
introduced by Carrier et al [10] and further generalized by Liñán and Williams [3], facilitates the treatment of the problem by allowing a self-similar description of the non-reactive scalars. In the development, the mixing-layer thickness is defined from the evolution problem

$$
\delta \frac{\mathrm{d} \delta}{\mathrm{d} t}=D_{\mathrm{T}}-a \delta^{2} \quad \delta(0)=\delta_{i}
$$

where $\delta_{i}$ is a characteristic value of $\delta$ at $t=0$ and $D_{\mathrm{T}}$ is the thermal diffusivity of the gas mixture. Clearly, the evolution of $\delta$ depends on the competition of strain and diffusion.

In terms of the scaled coordinate $\eta$, the species and energy conservation equations corresponding to a reactive system with $N$ different chemical species reduce to

$$
\begin{aligned}
& \frac{\partial Y_{i}}{\partial t}-\frac{D_{\mathrm{T}}}{\delta^{2}}\left(\eta \frac{\partial Y_{i}}{\partial \eta}+\frac{1}{L_{i}} \frac{\partial^{2} Y_{i}}{\partial \eta^{2}}\right)=\frac{\omega_{i}}{\rho} \\
& \frac{\partial T}{\partial t}-\frac{D_{\mathrm{T}}}{\delta^{2}}\left(\eta \frac{\partial T}{\partial \eta}+\frac{\partial^{2} T}{\partial \eta^{2}}\right)=-\sum_{i=1}^{N} \frac{h_{i}^{o} \omega_{i}}{\rho c_{p}}
\end{aligned}
$$

where $T$ is the temperature, and $Y_{i}, L_{i}=D_{\mathrm{T}} / D_{i}, D_{i}, \omega_{i}$ and $h_{i}^{o}$ denote, respectively, the mass fraction, Lewis number, Fickian diffusion coefficient, mass rate of production per unit volume and enthalpy of formation of species $i$. Constant values $L_{\mathrm{O}_{2}}=0.9, L_{\mathrm{H}_{2}}=0.3, L_{\mathrm{H}}=0.2$ and $L_{\mathrm{O}}=L_{\mathrm{OH}}=0.85$ are adopted for the different Lewis numbers in the computations presented below. According to (2) and (3), the relevant mechanical time in reactive mixing layers subject to variable strain is the diffusion time across the mixing layer $\delta^{2} / D_{\mathrm{T}}$ rather than the instantaneous strain time $a^{-1}$. Clearly, this characteristic becomes irrelevant in steady systems, for which $a=D_{\mathrm{T}} / \delta^{2}$ as indicated by (1).

Integration of (1)-(3) with a prescribed $a(t)$ determines the evolution of the reactive mixing layer. Appropriate boundary conditions for reactants and temperature corresponding to counterflowing streams of air and fuel are $Y_{\mathrm{O}_{2}}-Y_{\mathrm{O}_{2} \infty}=Y_{\mathrm{H}_{2}}=T-T_{\infty}=0$ at $\eta=+\infty$ and $Y_{\mathrm{O}_{2}}=Y_{\mathrm{H}_{2}}-Y_{\mathrm{H}_{2}-\infty}=T-T_{-\infty}=0$ at $\eta=-\infty$. On the other hand, initial conditions should be provided to define a well posed problem. For instance, the initial conditions corresponding to a sharp interface separating both sides of the mixing layer at $t=0$ are $Y_{\mathrm{O}_{2}}-Y_{\mathrm{O}_{2} \infty}=Y_{\mathrm{H}_{2}}=T-T_{\infty}=0$ for $\eta>0$ and $Y_{\mathrm{O}_{2}}=Y_{\mathrm{H}_{2}}-Y_{\mathrm{H}_{2}-\infty}=T-T_{-\infty}=0$ for $\eta<0$, together with $\delta=\delta_{i}=0$. It is assumed that the feed streams are radical free, so that $Y_{i}=0$ is the appropriate boundary conditions for all radical mass fractions at $\eta= \pm \infty$. Similarly, if no chemical reaction is considered before $t=0$, then the initial condition for all radicals is $Y_{i}=0$ everywhere across the mixing layer.

\subsection{Chemistry description}

Attention is restricted to initial temperatures sufficiently above crossover for the recombination reactions to be negligibly slow, a limit for which the chemistry description reduces to the initiation reaction

$$
\mathrm{H}_{2}+\mathrm{O}_{2} \stackrel{1}{\rightarrow} \mathrm{OH}+\mathrm{OH}
$$

the chain-branching reactions

$$
\begin{aligned}
& \mathrm{H}+\mathrm{O}_{2} \stackrel{2}{\rightarrow} \mathrm{OH}+\mathrm{O} \\
& \mathrm{O}+\mathrm{H}_{2} \stackrel{3}{\rightarrow} \mathrm{OH}+\mathrm{H}
\end{aligned}
$$

and the chain-carrying reaction

$$
\mathrm{OH}+\mathrm{H}_{2} \stackrel{4}{\rightarrow} \mathrm{H}_{2} \mathrm{O}+\mathrm{H} .
$$


The reaction-rate constants of the above chemical scheme depend on the temperature according to $k_{j}=A_{j} T^{n_{j}} \exp \left[-E_{j} /\left(R^{o} T\right)\right]$. Values of the reaction-rate parameters are given in $\mathrm{mol} \mathrm{cm}{ }^{-3}, \mathrm{~K}$ and cal mol${ }^{-1}$ by [11] $A_{1}=1.7 \times 10^{13}, A_{2}=3.52 \times 10^{16}, A_{3}=5.06 \times 10^{4}$, $A_{4}=1.17 \times 10^{9}, n_{1}=0, n_{2}=-0.7, n_{3}=2.67, n_{4}=1.3, E_{1}=47780, E_{2}=17070$, $E_{3}=6290$ and $E_{4}=3626$.

Since it is through radical recombination that most of the heat is released in $\mathrm{H}_{2}-\mathrm{O}_{2}$ combustion, at temperatures high above crossover the resulting chain-branching process is thermally frozen. Also, reactant consumption can be neglected in the first approximation to study the radical growth process [12], so that the temperature and reactant profiles can be obtained by integration of (2) and (3) with $\omega_{i}=0$. The associated chemically frozen profiles become time-independent after a characteristic time of order $\delta_{i}^{2} / D_{\mathrm{T}}$ and are then given by

$$
\begin{aligned}
& y_{\mathrm{O}_{2} f}=Y_{\mathrm{O}_{2} f} / Y_{\mathrm{O}_{2} \infty}=1-\frac{1}{2} \operatorname{erfc}\left[\left(L_{\mathrm{O}_{2}} / 2\right)^{1 / 2} \eta\right] \\
& y_{\mathrm{H}_{2} f}=Y_{\mathrm{H}_{2} f} / Y_{\mathrm{H}_{2}-\infty}=\frac{1}{2} \operatorname{erfc}\left[\left(L_{\mathrm{H}_{2}} / 2\right)^{1 / 2} \eta\right] \\
& \theta_{\mathrm{f}}=\left(T_{\mathrm{f}}-T_{\infty}\right) / T_{\infty}=\frac{\theta_{-\infty}}{2} \operatorname{erfc}\left[\left(\frac{1}{2}\right)^{1 / 2} \eta\right]
\end{aligned}
$$

where $\theta_{-\infty}=\left(T_{-\infty}-T_{\infty}\right) / T_{\infty}$ and erfc represents the complementary error function. Negative values of $\theta_{-\infty}$ are found in cases where the air-side temperature is larger than the hydrogen temperature, as occurs typically in propulsion applications.

\subsection{Radical conservation equations}

The problem then reduces to that of integrating the radical conservation equations

$$
\begin{aligned}
& \frac{\partial y_{\mathrm{H}}}{\partial \tau}-\frac{1}{\Delta(\tau)}\left(\eta \frac{\partial y_{\mathrm{H}}}{\partial \eta}+\frac{1}{L_{\mathrm{H}}} \frac{\partial^{2} y_{\mathrm{H}}}{\partial \eta^{2}}\right)=-\kappa_{2} y_{\mathrm{H}}+\gamma\left(\kappa_{3} y_{\mathrm{O}}+r \kappa_{4} y_{\mathrm{OH}}\right) \\
& \frac{\partial y_{\mathrm{O}}}{\partial \tau}-\frac{1}{\Delta(\tau)}\left(\eta \frac{\partial y_{\mathrm{O}}}{\partial \eta}+\frac{1}{L_{\mathrm{O}}} \frac{\partial^{2} y_{\mathrm{O}}}{\partial \eta^{2}}\right)=\kappa_{2} y_{\mathrm{H}}-\gamma \kappa_{3} y_{\mathrm{O}} \\
& \frac{\partial y_{\mathrm{OH}}}{\partial \tau}-\frac{1}{\Delta(\tau)}\left(\eta \frac{\partial y_{\mathrm{OH}}}{\partial \eta}+\frac{1}{L_{\mathrm{OH}}} \frac{\partial^{2} y_{\mathrm{OH}}}{\partial \eta^{2}}\right)=\kappa_{1}+\kappa_{2} y_{\mathrm{H}}+\gamma\left(\kappa_{3} y_{\mathrm{O}}-r \kappa_{4} y_{\mathrm{OH}}\right)
\end{aligned}
$$

with initial conditions

$$
y_{\mathrm{H}}(0, \eta)=y_{\mathrm{O}}(0, \eta)=y_{\mathrm{OH}}(0, \eta)=0
$$

and boundary conditions

$$
y_{\mathrm{H}}(\tau, \pm \infty)=y_{\mathrm{O}}(\tau, \pm \infty)=y_{\mathrm{OH}}(\tau, \pm \infty)=0 .
$$

The characteristic chemical time of reaction 2 evaluated at $T=T_{\infty},\left(\rho k_{2 \infty} Y_{\mathrm{O}_{2} \infty} / W_{\mathrm{O}_{2}}\right)^{-1}$, has been employed to define the normalized time $\tau=t\left(\rho k_{2 \infty} Y_{\mathrm{O}_{2} \infty} / W_{\mathrm{O}_{2}}\right)$, with $W_{i}$ representing in this notation the molecular weight of species $i$. This same chemical time has been used in defining the chain-branching Damköhler number $\Delta$ given below in (20).

On the other hand, the parameters

$$
\gamma=\frac{k_{3 \infty}}{k_{2 \infty}} \frac{W_{\mathrm{O}_{2}}}{W_{\mathrm{H}_{2}}} \frac{Y_{\mathrm{H}_{2}-\infty}}{Y_{\mathrm{O}_{2} \infty}}
$$


and

$$
r=\frac{k_{4 \infty}}{k_{3 \infty}}
$$

measure the ratios of the characteristic branching time of reaction 2 to that of reaction 3 and of the characteristic branching time of reaction 3 to that of reaction 4 . The functions of the order of unity

$$
\begin{aligned}
& \kappa_{1}(\eta)=\exp \left[\beta_{1} \theta_{\mathrm{f}} /\left(1+\theta_{\mathrm{f}}\right)\right] y_{\mathrm{O}_{2} f} y_{\mathrm{H}_{2} f} \\
& \kappa_{2}(\eta)=\exp \left[\beta_{2} \theta_{\mathrm{f}} /\left(1+\theta_{\mathrm{f}}\right)\right] y_{\mathrm{O}_{2} f} \\
& \kappa_{3}(\eta)=\exp \left[\beta_{3} \theta_{\mathrm{f}} /\left(1+\theta_{\mathrm{f}}\right)\right] y_{\mathrm{H}_{2} f}
\end{aligned}
$$

and

$$
\kappa_{4}(\eta)=\exp \left[\beta_{4} \theta_{\mathrm{f}} /\left(1+\theta_{\mathrm{f}}\right)\right] y_{\mathrm{H}_{2} f}
$$

represent reduced Damköhler numbers evaluated across the mixing layer with the local frozen values of the reactant mass fractions and temperature. For simplicity, the algebraic temperature dependences of the different reaction-rate constants have been incorporated here in the definition of the non-dimensional activation energies $\beta_{j}=E_{j} /\left(R^{o} T_{\infty}\right)+n_{j}$. The radical mass fractions

$$
\begin{aligned}
& y_{\mathrm{H}}=\frac{k_{2 \infty}}{2 k_{1 \infty}} \frac{W_{\mathrm{H}_{2}}}{W_{\mathrm{H}}} \frac{Y_{\mathrm{H}}}{Y_{\mathrm{H}_{2}-\infty}} \\
& y_{\mathrm{O}}=\frac{k_{2 \infty}}{2 k_{1 \infty}} \frac{W_{\mathrm{H}_{2}}}{W_{\mathrm{O}}} \frac{Y_{\mathrm{O}}}{Y_{\mathrm{H}_{2}-\infty}} \\
& y_{\mathrm{OH}}=\frac{k_{2 \infty}}{2 k_{1 \infty}} \frac{W_{\mathrm{H}_{2}}}{W_{\mathrm{OH}}} \frac{Y_{\mathrm{OH}}}{Y_{\mathrm{H}_{2}-\infty}}
\end{aligned}
$$

are scaled with the ratio of the branching time to the initiation time

$$
\nu=k_{1 \infty} / k_{2 \infty}
$$

a very small quantity that takes for instance the values $1.7 \times 10^{-7}$ and $4.3 \times 10^{-5}$ at $T_{\infty}=1200$ and $2000 \mathrm{~K}$, respectively. With this definition, $y_{\mathrm{H}}, y_{\mathrm{O}}$ and $y_{\mathrm{OH}}$ will be of the order of unity during an initial stage, corresponding to $\tau$ of the order of unity, in which the initiation reaction and the chain-branching reactions have contributions of the same order. The chain-branching growth described by (7)-(9) ends as the radical mole fractions reach values of the order of unity, leading through a relatively short adjustment period not studied here to a solution with partial equilibrium of reactions $2-4$. The ignition time can be therefore identified as the time for which the variables $y_{i}$ reach large values of order $v^{-1} \gg 1$ [12].

\section{Damköhler-number evolution}

Changes in the strain rate $a(t)$ influence radical growth by modifying the gradients of reactants and temperature in the mixing layer as well as the rate of radical loss by convection and diffusion. These effects are globally captured in (7)-(9) by the variable Damköhler number

$$
\Delta(\tau)=\left(\rho k_{2 \infty} Y_{\mathrm{O}_{2} \infty} / W_{\mathrm{O}_{2}}\right) /\left(D_{\mathrm{T}} / \delta^{2}\right)
$$


defined as the ratio of the diffusion time across the mixing layer $\delta^{2} / D_{\mathrm{T}}$ to the characteristic branching time $\left(\rho k_{2 \infty} Y_{\mathrm{O}_{2} \infty} / W_{\mathrm{O}_{2}}\right)^{-1}$. The evolution equation for this function follows from a straightforward extension of (1),

$$
\frac{1}{2} \frac{\mathrm{d} \Delta}{\mathrm{d} \tau}=1-\bar{a}(\tau) \Delta \quad \Delta(0)=\Delta_{i}
$$

where $\bar{a}=a /\left(\rho k_{2 \infty} Y_{\mathrm{O}_{2} \infty} / W_{\mathrm{O}_{2}}\right)$ and $\Delta_{i}=\left(\rho k_{2 \infty} Y_{\mathrm{O}_{2} \infty} / W_{\mathrm{O}_{2}}\right) /\left(D_{\mathrm{T}} / \delta_{i}^{2}\right)$. Separation of variables readily provides [3]

$\Delta(\tau)=\left[\Delta_{i}+2 \int_{0}^{\tau} \exp \left(2 \int_{0}^{\tau^{\prime}} \bar{a}\left(\tau^{\prime \prime}\right) \mathrm{d} \tau^{\prime \prime}\right) \mathrm{d} \tau^{\prime}\right] \exp \left(-2 \int_{0}^{\tau} \bar{a}\left(\tau^{\prime}\right) \mathrm{d} \tau^{\prime}\right)$

which can be used to evaluate $\Delta(\tau)$ for a given strain-rate history $\bar{a}(\tau)$.

It is worth investigating simple solutions of (22) in typical configurations of particular interest. For instance, for a constant strain rate $\bar{a}=\bar{a}_{0}$, the corresponding Damköhler-number function $\Delta(\tau)$ becomes

$$
\Delta=\bar{a}_{0}^{-1}+\left(\Delta_{i}-\bar{a}_{0}^{-1}\right) \exp \left(-2 \bar{a}_{0} \tau\right)
$$

revealing that the mixing layer adapts to the externally imposed strain rate after a time of order $a_{0}^{-1}$, i.e. only a weak dependence on the initial conditions remains in the solution for $\tau \gg \bar{a}_{0}^{-1}$. One can anticipate that, if $a$ changes slowly with a characteristic time larger than $a^{-1}$, then the mixing layer adapts to the instantaneous strain rate to give $\Delta=\bar{a}^{-1}$ (except possibly during a short initial period of order $a_{0}^{-1}$ ).

On the other hand, equation (22) gives $\Delta=2 \tau$ as the solution corresponding to an unstrained configuration. The associated form of (7)-(9) can then be used to investigate chainbranching growth in the isovelocity coflow mixing layer, with the time in (7)-(9) representing the downstream distance divided by the existing velocity [12]. In the work of Thévenin and Candel $[4,5]$, the ignition distance in the compressible plane shear layer that forms between two streams with different velocity was determined by extending the case of the isovelocity mixing layer in an approximate way. Thus, the evolution of a convected interface element was studied, and the problem is formulated as that of a time-dependent mixing layer with variable strain. The strain rate acting on the interface element was approximated as the ratio of the constant velocity difference between the two streams to the local shear-layer thickness, which is assumed to increase linearly with downstream distance [4]. Translating these ideas into the present formulation provides a variable strain rate $\bar{a}=\bar{a}_{0} /(1+c \tau)$, where the initial value $\bar{a}_{0}$ depends on the thickness of the splitter plate initially separating the streams and the nondimensional coefficient $c$ varies depending on the degree of compressibility [4]. Introducing this strain-rate law in (22) yields

$$
\Delta=\frac{2(1+c \tau)}{c+2 \bar{a}_{0}}
$$

for the Damköhler number behaviour at large times.

Another unsteady problem of interest is the ignition of a quiescent reactive gas by a hot emerging jet. Although the resulting unsteady problem is quite complex, one can anticipate that the onset of ignition is controlled by the evolution of combustion at the jet front, where a mixing layer subject to a decaying strain rate $a \propto t^{-1}$ forms. Correspondingly, the long-time behaviour of the associated Damköhler number can be anticipated to be of the form $\Delta \propto \tau$. Clearly, the above considerations suggest that the Damköhler-number function $\Delta(\tau)$ associated with many cases of practical interest is either a constant or a linearly increasing function of time (or a combination of both). 


\section{The steady-state assumptions for $\mathrm{O}$ and $\mathrm{OH}$}

With undiluted hydrogen assumed in the fuel stream, numerical evaluation of (12) and (13) yields for instance $\gamma \simeq 219$ and $r \simeq 4.3$ at $T=1200 \mathrm{~K}$ and $\gamma \simeq 200$ and $r=1.4$ at $T=2000 \mathrm{~K}$, respectively, indicating that reaction 2 is the slowest of the three branching reactions, and therefore becomes the rate-limiting step in typical applications, while the rates of reactions 3 and 4 are comparable in magnitude at the temperatures of interest. We therefore use the limit $\gamma \gg 1$ to simplify the chemistry description. In this limiting case the transport and accumulation rates of $\mathrm{O}$ and $\mathrm{OH}$ can be neglected in the first approximation, thereby yielding the steady-state expressions

$$
y_{\mathrm{O}}=\frac{\kappa_{2}}{\gamma \kappa_{3}} y_{\mathrm{H}}
$$

and

$$
y_{\mathrm{OH}}=\frac{\kappa_{1}}{\gamma r \kappa_{4}}+\frac{2 \kappa_{2}}{\gamma r \kappa_{4}} y_{\mathrm{H}}
$$

as replacements of (8) and (9). Introducing now (25) and (26) into the right-hand side of (7) provides

$$
\frac{\partial y_{\mathrm{H}}}{\partial \tau}-\frac{1}{\Delta(\tau)}\left[\eta \frac{\partial y_{\mathrm{H}}}{\partial \eta}+\frac{1}{L_{\mathrm{H}}} \frac{\partial^{2} y_{\mathrm{H}}}{\partial \eta^{2}}\right]=\kappa_{1}+2 \kappa_{2} y_{\mathrm{H}}
$$

for the evolution of the $\mathrm{H}$-atom mass fraction.

To test the accuracy of the resulting description, the evolution with time of the peak value of $y_{\mathrm{H}}$ obtained by integration of (27) with initial and boundary conditions given in (10) and (11) is compared in figure 1 with integrations of the original equations (7)-(9) for the isothermal mixing layer and for values of $\gamma=200$ and 20. A Crank-Nicholson method was employed in these numerical integrations, as well as in those of (29) presented below. In both plots, $r=2.0$ and a constant Damköhler number $\Delta=4.0$ is used. As can be seen, for the values of $\gamma$ employed in the plots, the resulting errors are too large, and lead to ignition times with errors decreasing with $\gamma$ but exceeding $20 \%$ even for values of $\gamma$ as large as $\gamma=200$. Similar inaccuracies were previously observed when assuming steady states for $\mathrm{O}$ and $\mathrm{OH}$ in calculations of ignition distances in the coflow supersonic mixing layer [13] and also in calculations of critical conditions for ignition in the counterflow mixing layer [14].

The origin of these relatively large inaccuracies lies in the failure of the steady-state approximations for $\mathrm{O}$ and $\mathrm{OH}$ as fuel is depleted on the air side of the mixing layer, where reactions 3 and 4 can no longer proceed at a fast rate. As can be concluded from (8) and (9), failure of these steady-state assumptions occurs as $\gamma \kappa_{3}$ and $\gamma \kappa_{4}$ become of the order of unity, which occurs for the isothermal mixing layer at a transverse location given approximately by $\eta_{\mathrm{f}} \simeq\left(2 \ln \gamma / L_{\mathbf{H}_{2}}\right)^{1 / 2} \gg 1$. The radical growth rate emerging from (27) is largely determined by the peak value of the branching rate $2 \kappa_{2}$. In most configurations of interest $\theta_{-\infty} \leqslant 0$, and the maximum branching coefficient $2 \kappa_{2}=2$ is achieved at $\eta=\infty$, beyond the region of applicability of the steady-state assumptions. Consequently, the integration of (27) overpredicts the radical growth rate, an effect clearly exhibited by the results of figure 1 .

An effective procedure that makes use of the steady-state approximations for $O$ and $\mathrm{OH}$, and still provides accurate results, is developed now. In the procedure, the steady-state expressions (25) and (26) are implemented after eliminating the large rates of reactions 3 and 4 from (7)-(9), leading to a single evolution equation for the radical pool. The development 

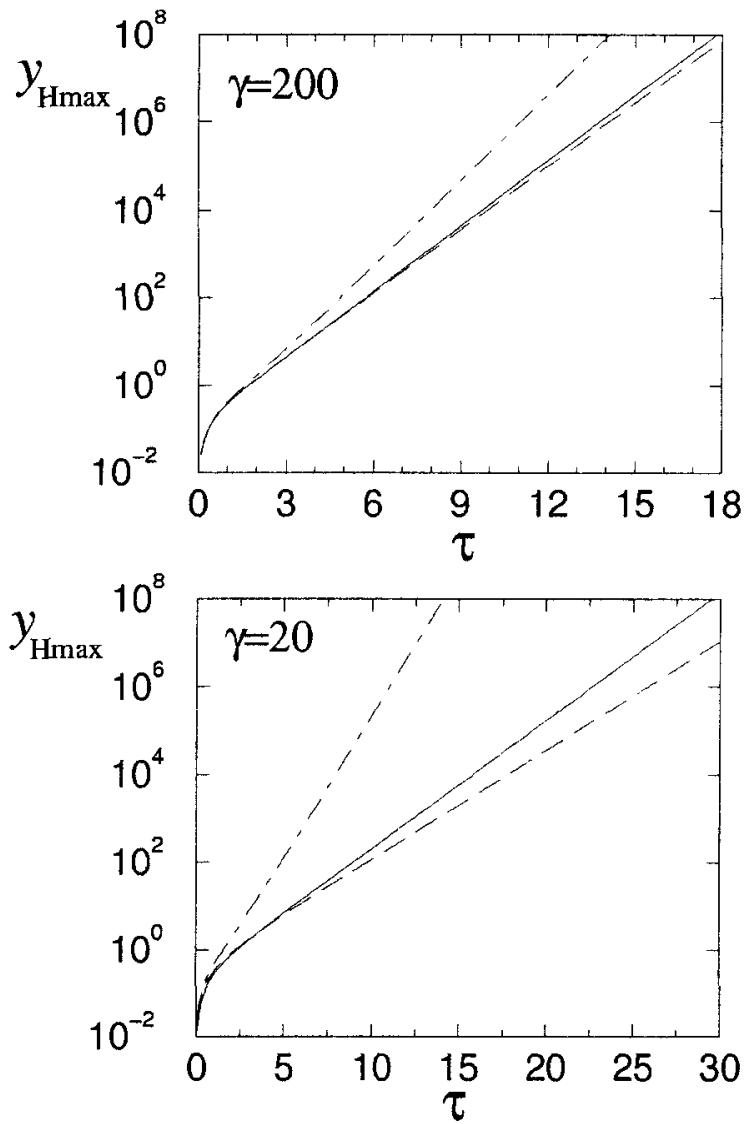

Figure 1. The evolution with time of the maximum value of $y_{H}$ obtained from (7)-(9) (full curve), from (27) (chain curve) and from (29) (broken curve) for the isothermal mixing layer with $r=2$ and $\Delta=4$.

therefore uses linear combinations of (7)-(9) to yield

$$
\begin{aligned}
& \frac{\partial}{\partial \tau}\left(y_{\mathrm{H}}+2 y_{\mathrm{O}}\right.\left.+y_{\mathrm{OH}}\right)-\frac{1}{\Delta(\tau)}\left[\eta \frac{\partial}{\partial \eta}\left(y_{\mathrm{H}}+2 y_{\mathrm{O}}+y_{\mathrm{OH}}\right)+\frac{\partial^{2}}{\partial \eta^{2}}\left(\frac{y_{\mathrm{H}}}{L_{\mathrm{H}}}+2 \frac{y_{\mathrm{O}}}{L_{\mathrm{O}}}+\frac{y_{\mathrm{OH}}}{L_{\mathrm{OH}}}\right)\right] \\
&=\kappa_{1}+2 \kappa_{2} y_{\mathrm{H}} .
\end{aligned}
$$

Then using (25) and (26), with the effect of initiation neglected in (26) (an approximation that applies strictly for $y_{\mathbf{H}} \gg 1$ ), yields

$$
\frac{\partial y}{\partial \tau}-\frac{1}{\Delta(\tau)}\left[\eta \frac{\partial y}{\partial \eta}+\frac{\partial^{2}}{\partial \eta^{2}}\left(\frac{y}{L}\right)\right]=\kappa_{1}+\kappa y
$$

for the evolution of the radical-pool variable

$$
y=y_{\mathrm{H}}+2 y_{\mathrm{O}}+y_{\mathrm{OH}}=\left[1+\frac{2 \kappa_{2}}{\gamma}\left(\frac{1}{\kappa_{3}}+\frac{1}{r \kappa_{4}}\right)\right] y_{\mathrm{H}} .
$$

In the formulation,

$$
L(\eta)=L_{\mathbf{H}} \frac{1+\left(2 \kappa_{2} / \gamma\right)\left(1 / \kappa_{3}+1 / r \kappa_{4}\right)}{1+\left(2 \kappa_{2} / \gamma\right)\left(L_{\mathrm{H}} / \kappa_{3} L_{\mathrm{O}}+L_{\mathrm{H}} / r \kappa_{4} L_{\mathrm{OH}}\right)}
$$


is an effective Lewis number and

$$
\kappa(\eta)=\frac{2 \kappa_{2}}{1+\left(2 \kappa_{2} / \gamma\right)\left(1 / \kappa_{3}+1 / r \kappa_{4}\right)}
$$

is the reduced chain-branching rate. Equation (29) must be integrated with initial and boundary conditions

$$
y(0, \eta)=0 \quad y(\tau, \pm \infty)=0
$$

as follows from (10) and (11).

Unlike the branching rate $2 \kappa_{2}$ appearing in (27), the function $\kappa(\eta)$ in (29) exhibits its maximum value at an intermediate transverse location $\eta^{*} \gg 1$, and decays monotonically on both sides, approaching a zero value at $\eta= \pm \infty$. To obtain the value of $\eta^{*}$, one can differentiate the simplified form of (32) for $\eta \gg 1$ and equate the resulting expression to zero, a procedure that yields. for instance, $\eta^{*} \simeq\left[2 \ln \gamma /\left(L_{\mathrm{H}_{2}}+L_{\mathrm{O}_{2}}\right)\right]^{1 / 2}$ for the isothermal mixing layer. Since $\eta_{\mathrm{f}} / \eta^{*}=\left[\left(L_{\mathrm{H}_{2}}+L_{\mathrm{O}_{2}}\right) / L_{\mathrm{H}_{2}}\right]^{1 / 2} \simeq 2$, the steady-state assumptions for $\mathrm{O}$ and $\mathrm{OH}$ hold at $\eta=\eta^{*}$, where the peak branching rate is reached, thereby enabling the simplified equation (29) to describe the radical growth history accurately. This is illustrated in figure 1 , where the evolution with time of the peak value of $y_{H}$ obtained from (30) after integration of (29) is included. Close agreement with the results of the original problem (7)-(9) is found even for a diluted fuel feed $(\gamma=20)$, thereby demonstrating that the steady-state assumptions for $\mathrm{O}$ and $\mathrm{OH}$ can be used to study the chain-branching explosion, provided that the resulting steady-state expressions are implemented as explained above.

\section{The branched-chain explosion}

The above results indicate that the radical-growth history in the mixing layer can be determined by integration of (29) with initial and boundary conditions given in (33), and with the function $\Delta(\tau)$ evaluated from (22) for a given strain rate $\bar{a}(\tau)$. The solution depends on the value of the temperature difference $\theta_{-\infty}$ and on the chemical-kinetic parameters $\gamma, r$ and $\beta_{i}$. Quasisteady solutions of the problem, addressed previously in a number of works $[6,7,8,14]$, correspond in the present formulation to a constant $\Delta$ with the accumulation term neglected in (29). Clearly, the value of $\Delta$ measures the competition of chemical production and radical loss by convection and diffusion. Thus, for values of $\Delta$ smaller than a critical value of the order of unity $\Delta_{c}$, the steady solutions are only weakly reactive, having radical mole fractions of order $v$ (values of $y$ of the order of unity) $[6,7,8,14]$. Values of $\Delta_{c}$ obtained from (29) for the conditions of figure 1 are $\Delta_{c}=0.90$ for $\gamma=200$ and $\Delta_{c}=1.37$ for $\gamma=20$, while the corresponding values obtained from the original equations (7)-(9) are $\Delta_{\mathfrak{c}}=0.86$ and $\Delta_{\mathfrak{c}}=1.19$, respectively.

With radical loss being dominant for $\Delta<\Delta_{\mathfrak{c}}$, the evolution of the radical pool from $y=0$ obtained by integrating the time-dependent equation (29) exhibits no chain-branching explosion when $\Delta(\tau)$ remains predominantly below $\Delta_{\mathrm{c}}$. On the other hand, previous numerical $[6,14]$ and analytical $[7,8]$ investigations of steady solutions revealed that the quasifrozen-type solutions no longer exist for $\Delta>\Delta_{\mathfrak{c}}$, being replaced by a branch of very reactive solutions with radical mole fractions of the order of unity (corresponding to values of $y$ of order $v^{-1}$ in the present formulation). Description of the resulting flames requires consideration of reactant consumption and of a more complicated chemistry description than that employed here. Since these additional effects are neglected in our analysis, no steady solution of (29) exists for $\Delta>\Delta_{c}$, and the associated time-dependent chain-branching explosion proceeds continuously with no upper bound to the value of $y$, a behaviour seen for instance in figure 1 . Nevertheless, equation (29) remains valid until ignition is completed when $y$ reaches a value of order $v^{-1}$, which occurs for values of $\tau \sim \ln \left(v^{-1}\right)$. 


\subsection{Solution for constant $\Delta$}

Numerical integration is required, in general, to determine the ignition history for a given strain rate $a(t)$, as done for instance in $[4,5]$ with an Arrhenius one-step reaction model adopted for the chemistry description. An exact solution to (29) can be found by separation of variables in cases where the Damköhler-number variation is negligible during the chainbranching explosion. Introducing

$$
y=\sum_{n=0}^{\infty} \alpha_{n} T_{n}(\tau) \phi_{n}(\eta)
$$

into (29) and taking into account that the initiation term is expressible in the form $\kappa_{1}(\eta)=$ $\sum_{n=0}^{\infty} \alpha_{n} \phi_{n}(\eta)$ provides the problems

$$
\frac{\mathrm{d} T_{n}}{\mathrm{~d} \tau}-\lambda_{n} T_{n}=1 \quad T_{n}(0)=0
$$

and

$$
\frac{\mathrm{d}^{2}}{\mathrm{~d} \eta^{2}}\left(\frac{\phi_{n}}{L(\eta)}\right)+\eta \frac{\mathrm{d} \phi_{n}}{\mathrm{~d} \eta}+\Delta\left(\kappa(\eta)-\lambda_{n}\right) \phi_{n}=0 \quad \phi_{n}( \pm \infty)=0 .
$$

The first equation can be readily integrated to give

$$
T=\frac{1}{\lambda_{n}}\left[\exp \left(\lambda_{n} \tau\right)-1\right]
$$

The exponential growing rate, $\lambda_{n}$, and the shape $\phi_{n}(\eta)$ of the different modes are the eigenvalues and corresponding eigenfunctions of the Sturm-Liouville problem (36), whereas imposing the condition $\kappa_{1}(\eta)=\sum_{n=0}^{\infty} \alpha_{n} \phi_{n}(\eta)$ provides

$$
\alpha_{n}=\frac{\int_{-\infty}^{\infty} \exp \left[\int_{0}^{\eta} L \bar{\eta} \mathrm{d} \bar{\eta}\right] \kappa_{1} \phi_{n} \mathrm{~d} \eta}{\int_{-\infty}^{\infty} \exp \left[\int_{0}^{\eta} L \bar{\eta} \mathrm{d} \bar{\eta}\right] \phi_{n}^{2} / L \mathrm{~d} \eta}
$$

for constant coefficients of the order of unity $\alpha_{n}$.

For a given value of $\Delta$, solution to (36) can be found for a discrete set of real values of $\lambda_{n}=\lambda_{0}, \lambda_{1}, \lambda_{2}, \ldots$, such that $\lambda_{0}>\lambda_{1}>\lambda_{2}>\ldots$. The eigenfunction $\phi=\phi_{0}$ associated with the largest eigenvalue $\lambda=\lambda_{0}$ has no zeros, while the number of zeros of the remaining eigenfunctions $\phi_{n}$ equals $n$. The values of $\lambda_{n}$ are increasing functions of $\Delta$, as can be seen in figure 2, where the variation with $\Delta$ of the three largest eigenvalues is plotted for the isothermal mixing layer with $\gamma=200$ and $r=2$. As previously explained, for $\Delta<\Delta_{c}$ radical loss dominates the branching process, and all the resulting eigenvalues are negative. On the other hand, for values of $\Delta$ larger than $\Delta_{c}$ at least one of the modes grows in time. Clearly, as $y$ increases for values of $\tau \gg 1$, the first mode dominates the solution, so that

$$
y=\left(\alpha_{0} / \lambda_{0}\right) \exp \left(\lambda_{0} \tau\right) \phi_{0}(\eta)
$$

becomes the leading-order representation of the radical pool.

\subsection{Ignition time}

As previously explained, the ignition time $t_{i}$ can be identified as the time for which the radical mass fractions achieve values of the order of unity, which corresponds to values of the radicalpool variable $y$ of order $v^{-1}$. Solving (39) for $\tau$ with $y \gg 1$ yields in the first approximation 


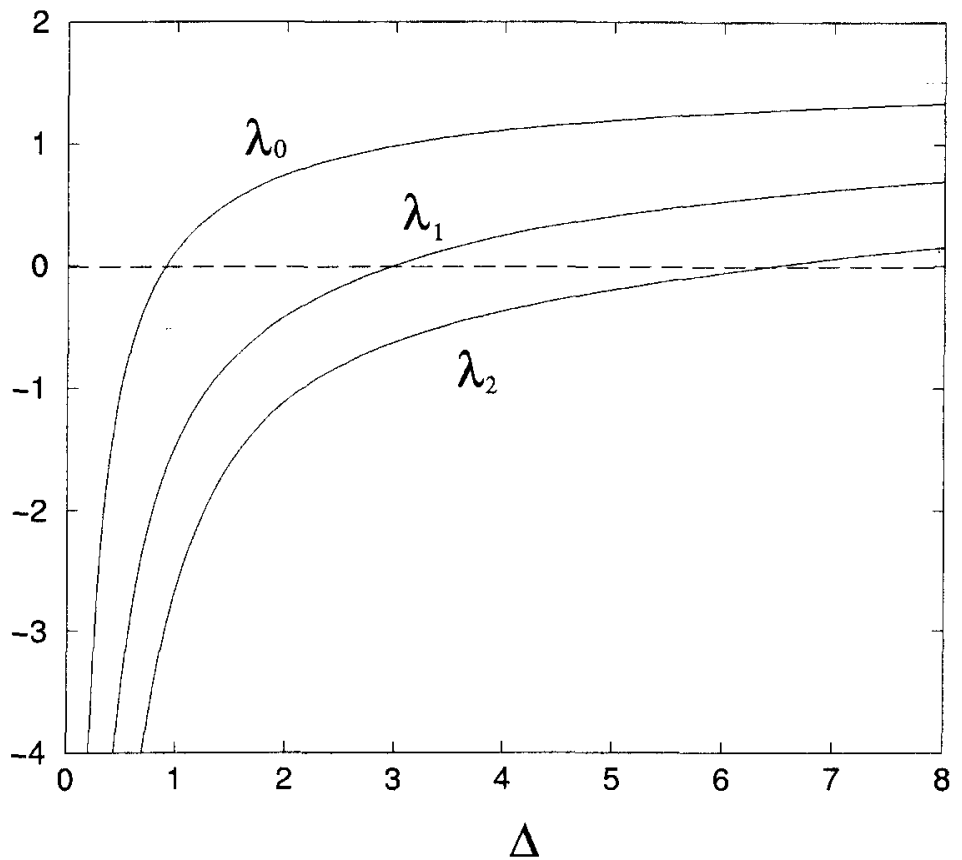

Figure 2. The variation with $\Delta$ of the first three eigenvalues of (36) for the isothermal mixing layer with $\gamma=200$ and $r=2$.

$\tau=\ln (y) / \lambda_{0}$. Evaluating this expression for $y=\nu^{-1}$ and using the definitions of $\tau$ and $\nu$ finally gives

$$
t_{i}=\left(\rho k_{2 \infty} Y_{\mathrm{O}_{2} \infty} / W_{\mathrm{O}_{2}}\right)^{-1} \ln \left(k_{2 \infty} / k_{1 \infty}\right) / \lambda_{0}
$$

as an equation to compute the ignition time $t_{i}$. Since the eigenvalue $\lambda_{0}$ enters in the computation of $t_{i}$, it is of interest to investigate its parametric dependence. Apart from the Lewis numbers, whose constant values are given below (3), seven different parameters enter in the solution of (36), namely, $\Delta, \gamma, r, \theta_{-\infty}, \beta_{2}, \beta_{3}$ and $\beta_{4}$.

The dependence of $\lambda_{0}$ can be simplified for the isothermal mixing layer, for which $\theta_{-\infty}=0$, thereby giving reduced reaction rates $\kappa_{2}=y_{\mathrm{O}_{2} f}, \kappa_{3}=y_{\mathrm{H}_{2} f}$ and $\kappa_{4}=y_{\mathrm{H}_{2} f}$ independent of the activation energies. Furthermore, since $\kappa_{3}=\kappa_{4}$ and with $L_{\mathrm{OH}}$ assumed to be approximately equal to $L_{O}$, the parameters $\gamma$ and $r$, which enter through the functions $\kappa(\eta)$ and $L(\eta)$, can be grouped into the single parameter

$$
\bar{\gamma}=\frac{\gamma r}{1+r}=\frac{k_{3 \infty} k_{4 \infty}}{k_{2 \infty}\left(k_{3 \infty}+k_{4 \infty}\right)} \frac{W_{\mathrm{O}_{2}}}{W_{\mathrm{H}_{2}}} \frac{Y_{\mathrm{H}_{2}-\infty}}{Y_{\mathrm{O}_{2} \infty}} .
$$

The variation of $\lambda_{0}$ with $\Delta$ for different values of $\bar{\gamma}$ was obtained from (36). In the numerical integration, an equilibrium finite-difference method was employed, with a secondorder centred-difference approximation adopted for the differential operators. The results are presented in figure 3, along with the asymptotic prediction for $\Delta \gg 1$ to be developed below.

In propulsion applications, the fuel stream is typically colder than the air stream, thereby causing the temperature in the reaction region to be lower than $T_{\infty}$. This effect is investigated in figure 4 , where the variation of $\lambda_{0}$ with $\Delta$ for negative values of $\theta_{-\infty}$ is compared with the results of the isothermal mixing layer for an undiluted fuel feed $(\gamma=200)$. As can be seen, 


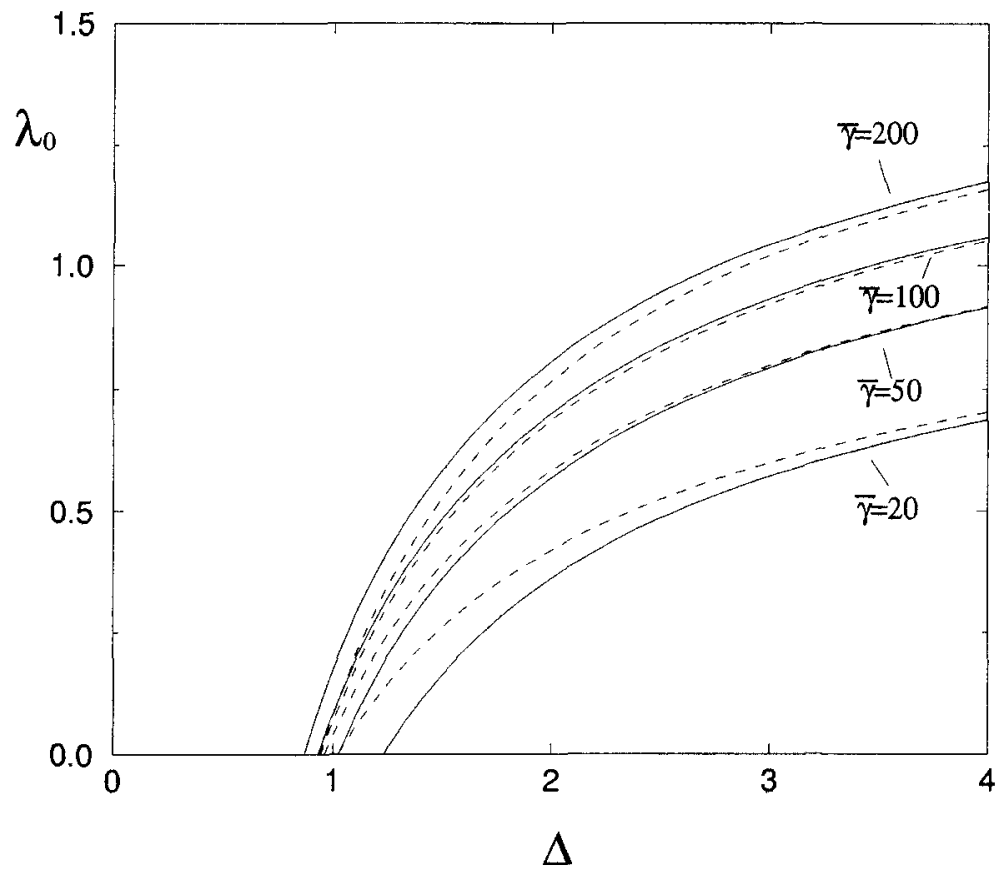

Figure 3. The variation of $\lambda_{0}$ with $\Delta$ for the isothermal mixing layer as obtained from (36) (full curves) and from the asymptotic expansion (49) (broken curves).

since the chemical activity occurs mainly on the oxidizer side of the mixing layer, the fuel-side temperature exerts only a limited influence on the rate of branching. It is worth pointing out that the parameters $r, \beta_{2}, \beta_{3}$ and $\beta_{4}$ have a non-negligible dependence on the value of $T_{\infty}$. While the parametric values used in the calculations $\left(r=2.52, \beta_{2}=5.02, \beta_{3}=4.78\right.$ and $\left.\beta_{4}=2.51\right)$ were evaluated for an air-side temperature $T_{\infty}=1500 \mathrm{~K}$, computations with different values of these four parameters corresponding to $T_{\infty}$ in the range $1200 \mathrm{~K}<T_{\infty}<2000 \mathrm{~K}$ were also performed. For a given value of the temperature difference $\theta_{-\infty}$, the resulting curve $\lambda_{0}(\Delta)$ showed only small departures from the corresponding curve in figure 4, indicating that for an undiluted fuel feed the exponential growth rate $\lambda_{0}$ depends only on the values of $\Delta$ and $\theta_{-\infty}$. Therefore, figure 4 can be used, in general, to calculate $\lambda_{0}$ irrespective of the value of $T_{\infty}$.

\subsection{Slowly varying strain}

An extension of the above analysis to cover cases with variable strain can be readily performed when the characteristic time of strain-rate variation is larger than the characteristic branching time, thereby yielding a slowly evolving Damköhler-number function $\Delta(\tau)=\bar{a}^{-1}$. In the proposed simplified description, both the shape and the growth rate of the radical pool are assumed to respond in a quasisteady fashion to variations of strain, so that (35) and (36) remain valid in the first approximation. However, the slow variation of the resulting exponential growth rate $\lambda_{0}(\tau)$ must be taken into account when integrating (35), thereby yielding

$$
y=\left(\alpha_{0} / \lambda_{0}\right) \exp \left(\int_{0}^{\tau} \lambda_{0} \mathrm{~d} \tau\right) \phi_{0}(\eta)
$$




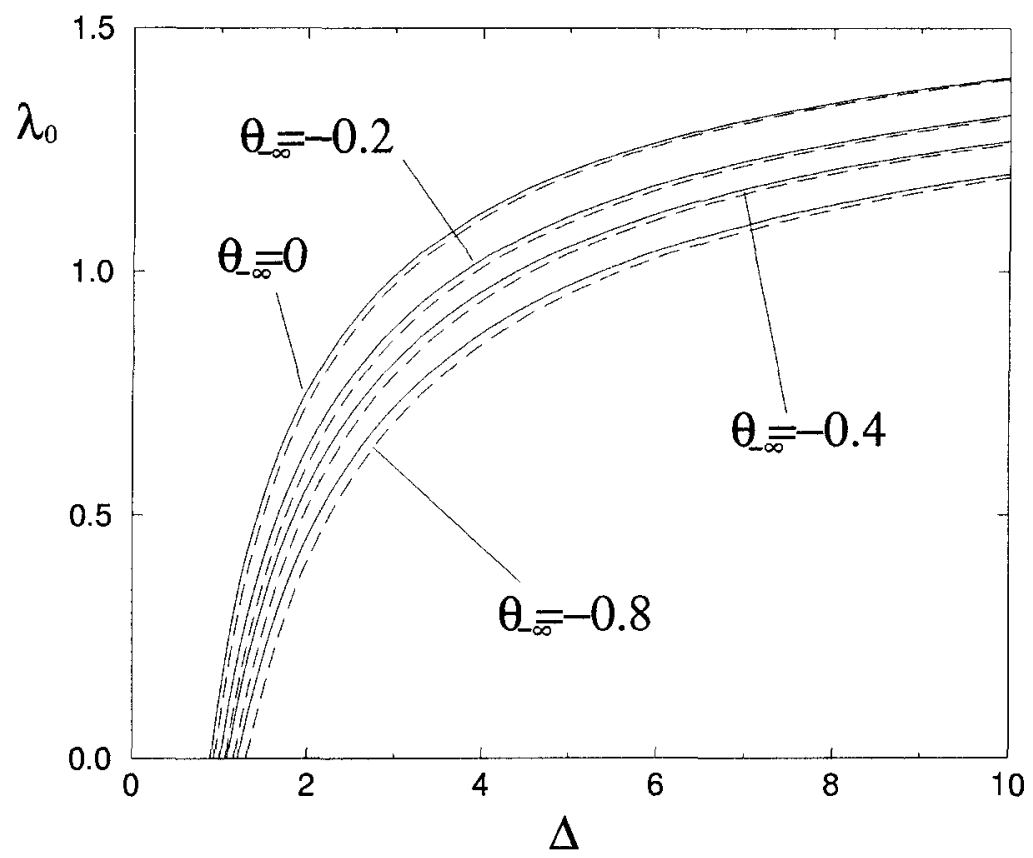

Figure 4. The variation of $\lambda_{0}$ with $\Delta$ for different values of $\theta_{-\infty}$ as obtained from (36) (full curves) and from the asymptotic expansion (49) (broken curves) with $\gamma=200, r=2.52, \beta_{2}=5.02$, $\beta_{3}=4.78$ and $\beta_{4}=2.51$.

as the leading-order representation for the radical pool. As before, the functions $\lambda_{0}(\tau)$ and $\phi_{0}(\eta)$ are the largest eigenvalue and corresponding eigenfunction of the eigenvalue problem (36) with $\Delta=\bar{a}^{-1}$.

The approximate description given in (42) gives errors of the order of unity, $\exp \left(\int_{0}^{\tau} \lambda_{0} \mathrm{~d} \tau\right)$, for the evolution of the peak radical mass fraction. Correspondingly, the dimensionless ignition time, $\tau_{i}$, is given implicitly by

$$
\int_{0}^{\tau_{i}} \lambda_{0} \mathrm{~d} \tau=\ln \left(v^{-1}\right)
$$

The prediction $y_{\max }=\exp \left(\int_{0}^{\tau} \lambda_{0} \mathrm{~d} \tau\right)$ is compared in figure 5 with the peak value of $y$ obtained by integration of (29). In this first test, an arbitrary sinusoidal time variation $\Delta=3-2.5 \sin (2 \pi \tau / 10)$ is assumed for the Damköhler number. An isothermal mixing layer with $\gamma=200$ and $r=2$ is considered. Correspondingly, the upper curve in figure 2 was utilized to compute the variation of the eigenvalue $\lambda_{0}$ as a function of the instantaneous Damköhler number $\Delta$. Although the conditions corresponding to this oscillatory strain rate are predominantly supercritical $\left(\Delta>\Delta_{\mathrm{c}}=0.9\right)$, the amplitude of the resulting oscillations is large enough to provide subcritical periods with $\Delta<\Delta_{\mathrm{c}}$ during which radical loss predominates. This effect is reproduced by the quasisteady approximation, with the associated prediction for the peak radical mass fraction $y \simeq \exp \left(\int_{0}^{\tau} \lambda_{0} d \tau\right)$ closely following the time variation obtained from (29).

As a second test, we investigate the branched-chain explosion associated with the Damköhler-number evolution given in (24), which approximately describes the strain rate exerted on a fluid particle in a plane shear flow [4]. Quasisteady behaviours are observed when 


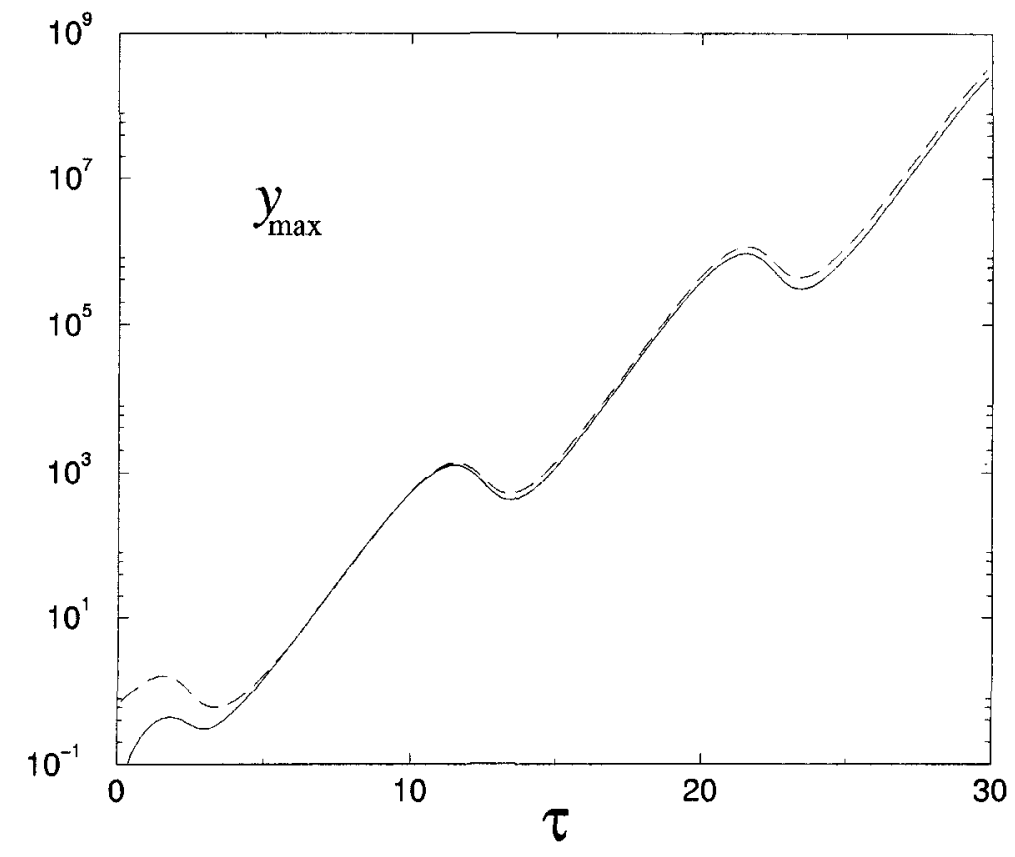

Figure 5. Comparison of the evolution with time of the maximum value of $y$ obtained from integration of (29) (full curve) with the quasisteady prediction $y_{\max }=\exp \left(\int_{0}^{\tau} \lambda_{0} \mathrm{~d} \tau\right)$ (broken curve) for the isothermal mixing layer with $\gamma=200, r=2$ and $\Delta=3-2.5 \sin (2 \pi \tau / 10)$.

the coefficients $\bar{a}_{0}$ and $c$ satisfy $c / \bar{a}_{0} \ll 1$, thereby reducing $(24)$ to $\Delta=\bar{a}^{-1}=(1+c \tau) / \bar{a}_{0}$. This condition is satisfied, for instance, in the experiment of Burrows and Kurkov [16] reported in [4]. Explicit expressions for calculating $\bar{a}_{0}=\left(V_{\mathrm{a}}-V_{\mathrm{f}}\right) W_{\mathrm{O}_{2}} /\left(\rho k_{2 \infty} Y_{\mathrm{O}_{2} \infty} \delta_{0}\right)$ and $c=\left[0.009\left(V_{\mathrm{a}}+V_{\mathrm{f}}\right)+0.151\left(V_{\mathrm{a}}-V_{\mathrm{f}}\right)\right] W_{\mathrm{O}_{2}} /\left(2 \rho k_{2 \infty} Y_{\mathrm{O}_{2} \infty} \delta_{0}\right)$ in configurations with negligible compressibility effects (as is the case for the experimental conditions in [16]) can be extracted from [4] as functions of the velocities of the air and fuel streams, $V_{\mathrm{a}}$ and $V_{\mathrm{f}}$, and of the thickness of the splitter plate separating the streams, $\delta_{0}$. Evaluating these expressions for the conditions of the experiment $\left(V_{\mathrm{a}}=1764, V_{\mathrm{f}}=1216 \mathrm{~m} \mathrm{~s}^{-1}, \delta_{0}=7.6 \times 10^{-4} \mathrm{~m}, T_{\infty}=1270, T_{-\infty}=254 \mathrm{~K}\right.$, $\left.Y_{\mathrm{O}_{2} \infty}=0.258, Y_{\mathrm{H}_{2}-\infty}=1.0\right)$ and with an average value $\rho=\left(\rho_{\infty}+\rho_{-\infty}\right) / 2=0.19 \mathrm{~kg} \mathrm{~m}^{-3}$ used for the density yields $\bar{a}_{0}=1.71$ and $c=0.171$.

The evolution of the maximum value of $y$ obtained from (29) for the Damköhler number $\Delta=(1+c \tau) / \bar{a}_{0}=0.584+0.1 \tau$ corresponding to the experiment of Burrows and Kurkov is plotted in figure 6 , along with the quasisteady prediction $\exp \left(\int_{0}^{\tau} \lambda_{0} \mathrm{~d} \tau\right)$. In the integration of (29), the values $\theta_{-\infty}=-0.8, \gamma=183.8, r=3.71, \beta_{1}=18.92, \beta_{2}=6.06, \beta_{3}=5.16$ and $\beta_{4}=2.73$ were employed (as corresponds approximately to the experimental conditions), while the bottom curve in figure 4 was used in the numerical evaluation of the integral $\int_{0}^{\tau} \lambda_{0} \mathrm{~d} \tau=\left(\bar{a}_{0} / c\right) \int_{\bar{a}_{0}^{-1}}^{\Delta} \lambda_{0} \mathrm{~d} \Delta$. As can be seen, the agreement between both approaches is very good for large times. Because of the influence of the initiation chemistry and of the higher modes on the solution, significant departures are observed, however, during the early development.

Ignition is reached in this configuration when $y=v^{-1} \simeq 10^{7}$, a criterion that determines the ignition time $\tau_{i}$. On the other hand, the time $\tau$ in figure 6 can be converted into a mixing-layer downstream distance $d=\tau\left(V_{\mathrm{a}}+V_{\mathrm{f}}\right) W_{\mathrm{O}_{2}} /\left(2 \rho k_{2 \infty} Y_{\mathrm{O}_{2} \infty}\right)$ by assuming that the 


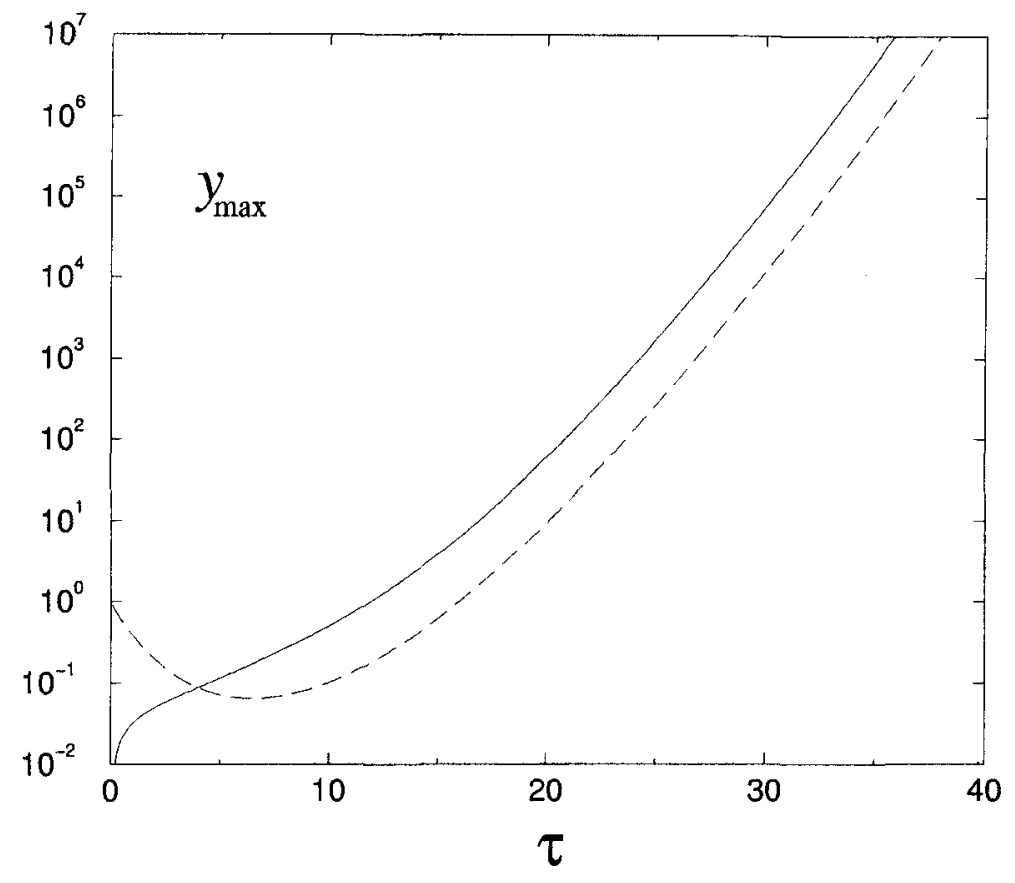

Figure 6. The evolution with time of the maximum value of $y$ for $\Delta=0.584+0.1 \tau$ as obtained from integration of (29) with $\theta_{-\infty}=-0.8, \gamma=183.8, r=3.71, \beta_{1}=18.92, \beta_{2}=6.06$, $\beta_{3}=5.16$ and $\beta_{4}=2.73$ (full curve) and from the quasisteady prediction $y_{\max }=\exp \left(\int_{0}^{\tau} \lambda_{0} \mathrm{~d} \tau\right)$ with $\lambda_{0}$ evaluated from figure 4 (broken curve).

reactive interface moves with the mean velocity $\left(V_{\mathrm{a}}+V_{\mathrm{f}}\right) / 2$ [4]. Using this expression yields $d_{i}=12.6 \mathrm{~cm}$ for the value $\tau_{i}=35.7$ obtained from the numerical integration of (29) and $d_{i}=13.4 \mathrm{~cm}$ for the quasisteady prediction $\tau_{i}=37.9$ corresponding to (43). Both results are in agreement with the experimental results, which suggested that the ignition distance lies in the range $\left(11 \mathrm{~cm}<d_{i}<15 \mathrm{~cm}\right)$ [4]. This test, as well as that presented in figure 5, demonstrates therefore how the quasisteady first-mode eigenvalues calculated as a function of $\Delta$ in figures 3 and 4 can be used in obtaining estimates of ignition times through (43) in mixing layers subjected to variable strain, providing the strain-rate variation is sufficiently slow. Furthermore, the second test validates the use of the time-dependent strain-rate formulation of Thévenin and Candel [4] in predicting chain-branching ignition distances in mixing layers.

\section{Ignition under weak strain}

To investigate further the effect of strain on radical growth we consider now the limit $\Delta(\tau) \gg 1$, corresponding to weakly strained mixing layers. This regime is amenable to an analytic description valid for constant $\Delta$ and also for cases with time-dependent strain such that the resulting Damköhler-number variation $\mathrm{d} \Delta / \mathrm{d} \tau$ can be expressed as an expansion in decreasing powers of $\Delta$. In particular, the analysis assumes a constant leading-order term of the order of unity in such an expansion, as corresponds for instance to the mixing layers discussed above after (22). Stronger leading-order variations of $\mathrm{d} \Delta / \mathrm{d} \tau$ of order $\Delta^{1 / 2}$ could also be accommodated in the asymptotic procedure, but such an analysis is not pursued further in this paper. 
The asymptotic analysis, which is developed in an appendix, yields

$$
\begin{gathered}
y=\frac{\sqrt{2} \kappa_{1}^{*}}{\kappa^{*}}\left[\exp \left(\Omega_{0} \tau+\Omega_{1} \int_{0}^{\tau} \Delta^{-1 / 2} \mathrm{~d} \tau+\Omega_{2} \int_{0}^{\tau} \Delta^{-1} \mathrm{~d} \tau\right)-1\right] \\
\quad \times \exp \left[-\left(-L^{*} \kappa^{\prime \prime} / 8\right)^{1 / 2} \Delta^{1 / 2}\left(\eta-\eta^{*}\right)^{2}\right]
\end{gathered}
$$

for the leading-order radical-pool representation, where the star $*$ refers to functions evaluated at $\eta=\eta^{*}$ and the primes ' denote derivatives with respect to $\eta$ evaluated also at $\eta=\eta^{*}$. The constant coefficients

$$
\begin{aligned}
& \Omega_{0}=\kappa^{*} \\
& \Omega_{1}=-\sqrt{-\kappa^{\prime \prime} /\left(2 L^{*}\right)}
\end{aligned}
$$

and

$$
\Omega_{2}=\frac{L^{*}}{4}\left(\frac{1}{L}\right)^{\prime} F_{1}+\frac{L^{*} \eta^{*}}{2} F_{2}-\frac{1}{4} \frac{\kappa^{\prime \prime \prime}}{\kappa^{\prime \prime}} F_{3}+F_{4}+\frac{1}{8} \frac{\mathrm{d} \Delta}{\mathrm{d} \tau}
$$

with

$$
\begin{aligned}
& F_{1}=-\frac{5}{12} \frac{\kappa^{\prime \prime \prime}}{\kappa^{\prime \prime}} \frac{1}{L^{*}}-\frac{1}{4}\left(\frac{1}{L}\right)^{\prime}-\frac{\eta^{*}}{2} \\
& F_{2}=-\frac{1}{4} \frac{\kappa^{\prime \prime \prime}}{\kappa^{\prime \prime}} \frac{1}{L^{*}}-\frac{3}{4}\left(\frac{1}{L}\right)^{\prime}-\frac{\eta^{*}}{2} \\
& F_{3}=-\frac{11}{36} \frac{\kappa^{\prime \prime \prime}}{\kappa^{\prime \prime}} \frac{1}{L^{*}}-\frac{7}{12}\left(\frac{1}{L}\right)^{\prime}-\frac{\eta^{*}}{2} \\
& F_{4}=-\frac{1}{16} \frac{\kappa^{\prime \prime \prime \prime}}{\kappa^{\prime \prime}} \frac{1}{L^{*}}+\frac{1}{8}\left(\frac{1}{L}\right)^{\prime \prime}-\frac{1}{2}
\end{aligned}
$$

can be computed from the functions $\kappa$ and $L$ and their derivatives. As can be seen in the last exponential term of (44), radicals are confined to a thin layer of relative thickness $\Delta^{-1 / 4}$ centred at the transverse location where the branching rate is maximum. As in previous asymptotic analyses of chain-branching ignition in coflow mixing layers $[12,15]$, the leadingorder exponential growth rate, independent of radical diffusion, corresponds to the maximum branching rate across the mixing layer. The associated ignition delay time $\tau_{i} \simeq \ln \left(v^{-1}\right) / \kappa^{*}$ becomes independent of the strain rate in the first approximation, with the effect of strain introducing a relatively small additional delay of order $\Delta^{-1 / 2}$. This finding has implications on the ignition of diffusion flames in a turbulent flow field. One can anticipate from the present asymptotic analysis that, for turbulence to affect the ignition process significantly, the characteristic time scale of the turbulent flow field must be of the order of the branching time, thereby yielding values of $\Delta$ of the order of unity. This observation seems to be verified by the direct numerical simulations of chain-branching ignition reported in [17]. Since the turbulent time scale was chosen in all computations of the order of the ignition time, which is a factor of $\ln \left(v^{-1}\right)$ larger than the branching time, the ignition times were found to be almost independent of the turbulence intensity for all the cases investigated.

Radical transport enters only at second order in the expansion for $\lambda_{0}$, giving a negative contribution to the exponential growth rate that corresponds to radical loss from the location of maximum growth. The third term in the asymptotic expansion for the growth rate is included for increased accuracy in configurations with only moderately large values of $\Delta$. As can be seen, the variation of $\Delta$ with time modifies the radical peak value through the integral terms appearing in the first exponential of (44) and also through a relatively small correction 


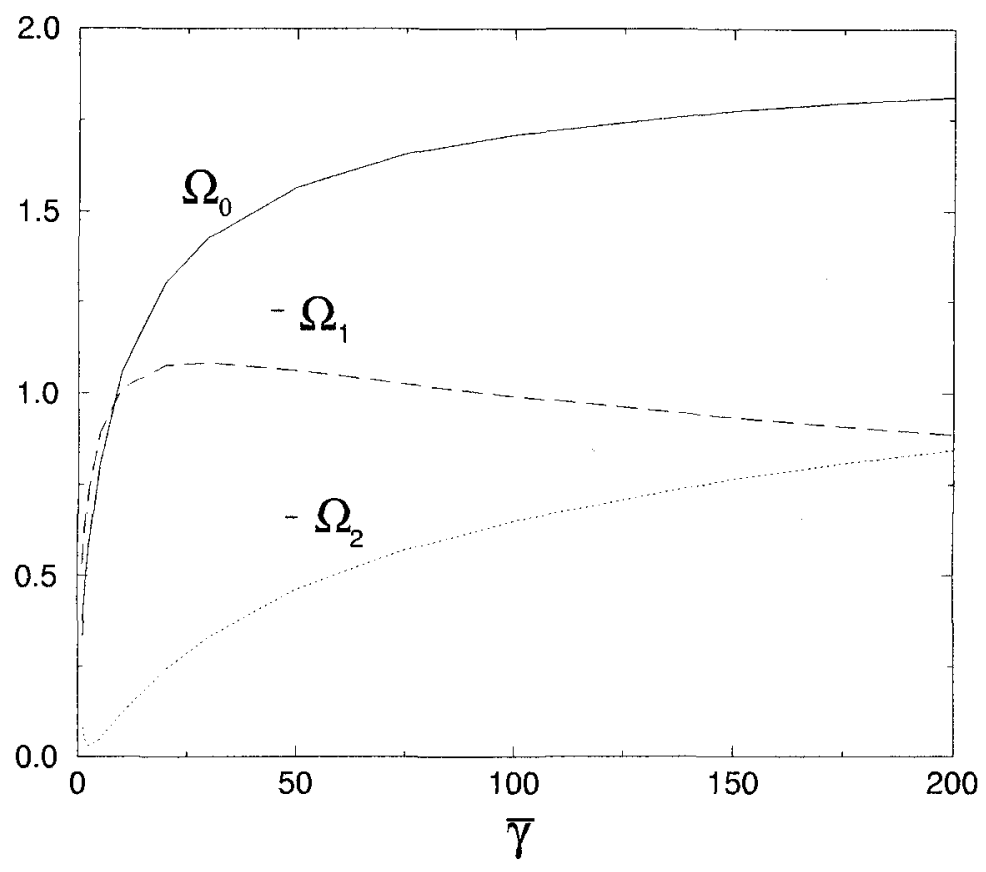

Figure 7. The variation with $\bar{\gamma}$ of the coefficients $\Omega_{0}, \Omega_{1}$ and $\Omega_{2}$ obtained from (45)-(48)

to the constant $\Omega_{2}$. On the other hand, the shape of the radical profile, determined by the last exponential factor in (44), evolves in a quasisteady fashion with a thickness that adapts instantaneously to the value of $\Delta$.

As expected, in configurations with constant $\Delta$, equation (44) reduces to (39). In this case, the asymptotic analysis yields in the first approximation

$$
\lambda_{0}=\Omega_{0}+\Delta^{-1 / 2} \Omega_{1}+\Delta^{-1} \Omega_{2}
$$

for the constant eigenvalue $\lambda_{0}$. This prediction is compared in figures 3 and 4 with the value of $\lambda_{0}$ determined from (36) for different conditions of dilution and fuel-side temperature. As can be seen, the three-term asymptotic expansion (49) produces excellent accuracy even for values of $\Delta$ of the order of unity. Because of its extended validity, (49) can be used in computations of ignition times in mixing layers with $\Delta \gtrsim 1$ as indicated in (40) and (43). To facilitate such computations, the values of the coefficients $\Omega_{0}, \Omega_{1}$ and $\Omega_{2}$ computed from (45)-(48) are given in figures 7 and 8 for different flow conditions of interest. In particular, figure 7 shows the variation of the coefficients with $\bar{\gamma}$ for the isothermal mixing layer, while their variation with $\theta_{-\infty}$ is given in figure 8 for the values of $\gamma, r$ and $\beta_{i}$ corresponding to figure 4 . Note that the values of $\Omega_{0}$ and $\Omega_{1}$ given in the plots are also valid for variable $\Delta$, while to calculate $\Omega_{2}$ one must add a correction $\frac{1}{8} \mathrm{~d} \Delta / \mathrm{d} \tau$ to the value given in figures 7 and 8 .

To illustrate the use of the expansion (49) to calculate ignition times, we consider again the variable Damköhler-number function (24) corresponding to the strain exerted on the interface of a plane shear layer, which reduces to $\Delta=\bar{a}^{-1}=(1+c \tau) / \bar{a}_{0}$ when $c / \bar{a}_{0} \ll 1$. In this case of quasisteady evolution the ignition time is determined from (43). Introducing (49) into (43) and using $\Delta$ as the integration variable finally gives

$$
\Omega_{0} c \tau_{i}+2 \Omega_{1} \bar{a}_{0}^{1 / 2}\left[\left(1+c \tau_{i}\right)^{1 / 2}-1\right]+\Omega_{2} \bar{a}_{0} \ln \left(1+c \tau_{i}\right)=c \ln \left(\nu^{-1}\right)
$$




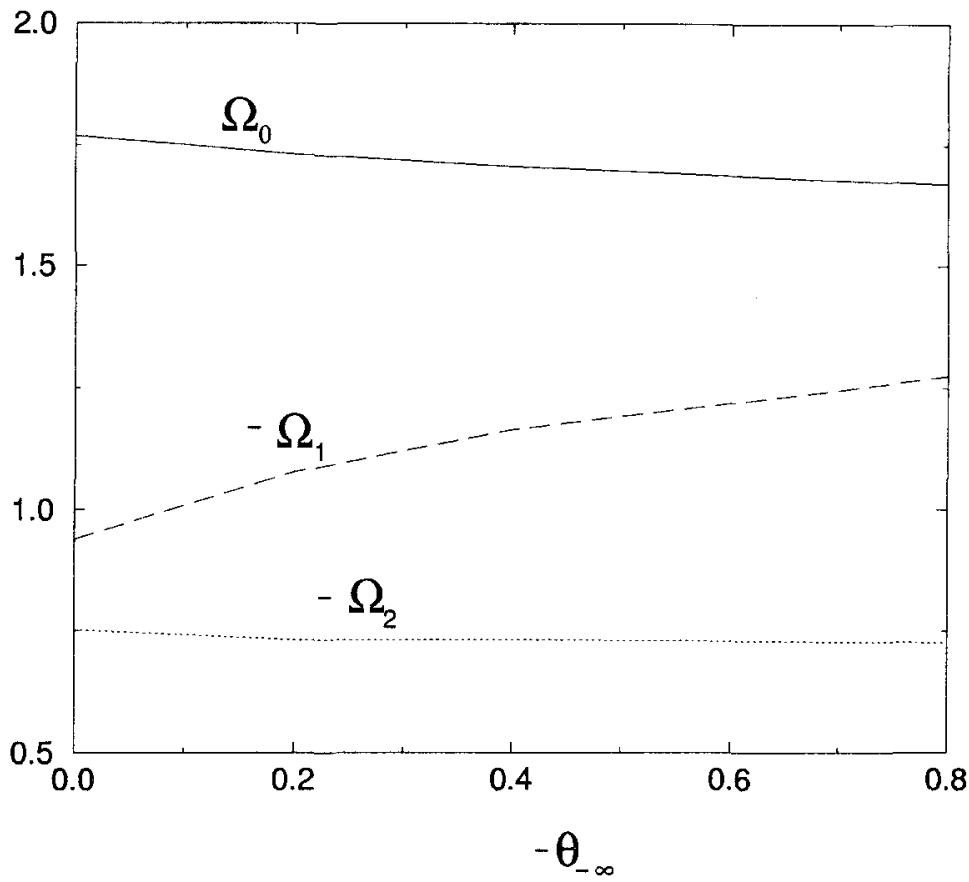

Figure 8. The variation with $\theta_{-\infty}$ of the coefficients $\Omega_{0}, \Omega_{1}$ and $\Omega_{2}$ obtained from (45)-(48) for $\gamma=200, r=2.52, \beta_{2}=5.02, \beta_{3}=4.78$ and $\beta_{4}=2.51$.

as an implicit equation for the ignition time, where $\Omega_{0}, \Omega_{1}$ and $\Omega_{2}$ can be extracted from figures 7 and 8 as previously indicated. For instance, for the experimental conditions of [16] $\bar{a}_{0}=1.71, c=0.171$ and $\nu \simeq 10^{-7}$, and the coefficients $\Omega_{0}=1.67, \Omega_{1}=-1.27$ and $\Omega_{2}=-0.73+c /\left(8 \bar{a}_{0}\right) \simeq-0.71$ can be obtained from figure 8 for $\theta_{-\infty}=-0.8$. Now solving (50) with these parametric values finally gives $\tau_{i}=39.05$, which corresponds to an ignition distance $d_{i}=13.78 \mathrm{~cm}$, again in agreement with the experimental results. If only one term had been retained in the expansion for $\lambda_{0}$, then the predictions for the ignition time and ignition distance would have been $\tau_{i}=9.65$ and $d_{i}=3.40 \mathrm{~cm}$, while a two-term expansion for $\lambda_{0}$ yields $\tau_{i}=24.62$ and $d_{i}=8.7 \mathrm{~cm}$. Clearly, in systems with only moderately large values of $\Delta$ or with values of $\Delta$ of the order of unity, retaining the three terms in the asymptotic expansion for $\lambda_{0}$ becomes a necessary complication to obtain accurate results.

It is remarkable that the general description (51) embodies as a particular case the solution in unstrained mixing layers, reproducing the results of alternative asymptotic methods $[12,15]$. When two stagnant half spaces, one of air and the other of hydrogen, are put into contact, the mixing layer that is formed grows in time giving a linearly increasing Damköhler number $\Delta=2 \tau$. The asymptotic description (44) therefore becomes applicable for large times. Introducing $\Delta=2 \tau$ into (44) and eliminating the spurious logarithmic singularity that appears at $\tau=0$, yielding

$y=\frac{\sqrt{2} \kappa_{1}^{*}}{\kappa^{*}} \exp \left[\Omega_{0} \tau+\sqrt{2} \Omega_{1} \tau^{1 / 2}+\Omega_{2} \ln (\tau) / 2\right] \exp \left[-\left(-L^{*} \kappa^{\prime \prime} / 4\right)^{1 / 2} \tau^{1 / 2}\left(\eta-\eta^{*}\right)^{2}\right]$

where the values of $\Omega_{0}$ and $\Omega_{1}$ are plotted in figures 7 and 8 , while $\Omega_{2}$ is obtained by adding $\frac{1}{8} \mathrm{~d} \Delta / \mathrm{d} \tau=\frac{1}{4}$ to the value given in the figures. The accuracy of (51) is tested in figure 9 , 


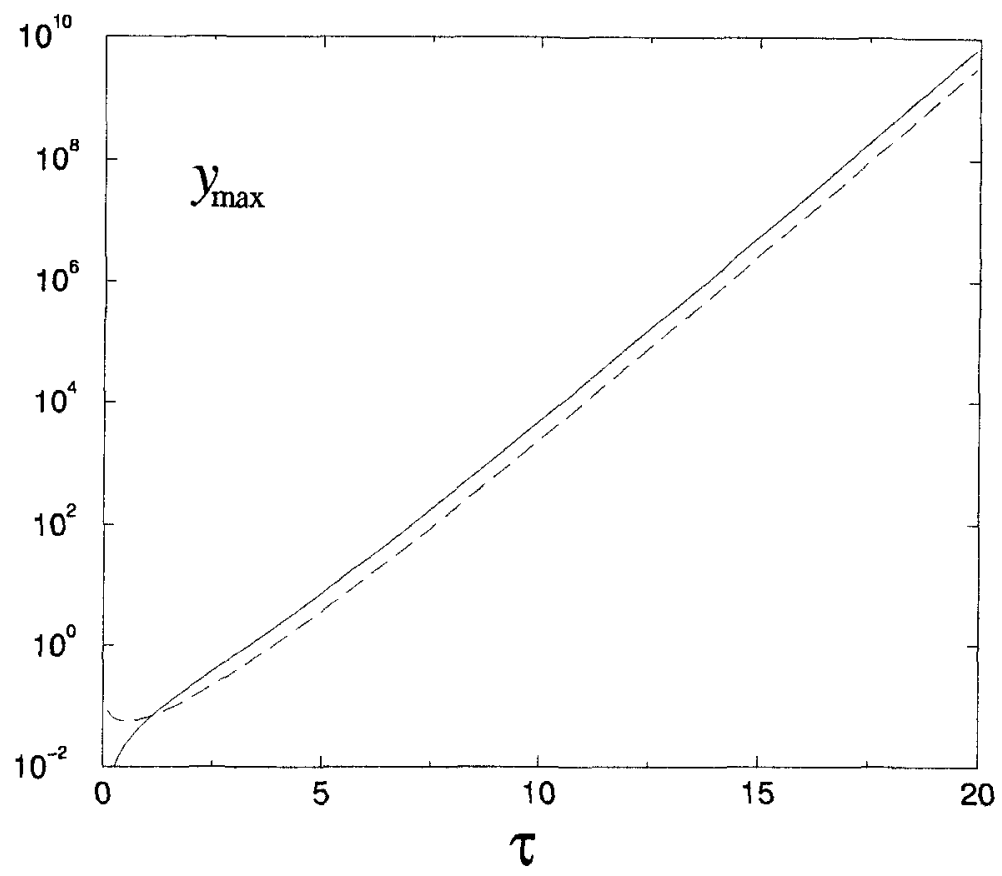

Figure 9. The time evolution of the maximum value of $y$ for the isovelocity coflow mixing layer with $\theta_{-\infty}=-0.8, \gamma=200, r=2.52, \beta_{2}=5.02, \beta_{3}=4.78$ and $\beta_{4}=2.51$ obtained from integration of (29) (full curve) and from evaluation of (51) (broken curve) with $\kappa_{1}^{*}=0.0851$, $\Omega_{0}=\kappa^{*}=1.67, \Omega_{1}=-1.27$ and $\Omega_{2}=-0.476$.

where the maximum value of $y$ obtained from integration of (29) with $\Delta=2 \tau, \theta_{-\infty}=-0.8$, $\gamma=200, r=2.52, \beta_{2}=5.02, \beta_{3}=4.78$ and $\beta_{4}=2.51$ is compared with the asymptotic prediction $y_{\max }=\left(\sqrt{2} \kappa_{1}^{*} / \kappa^{*}\right) \exp \left[\Omega_{0} \tau+\sqrt{2} \Omega_{1} \tau^{1 / 2}+\Omega_{2} \ln (\tau) / 2\right]$. As can be seen, because of the existence of an initiation-controlled initial period where the asymptotic description fails, errors of the order of unity remain as the solution develops for large times. Nevertheless, as indicated by the results in the plot, equation (51) can be expected to provide predictions of ignition times with errors smaller than 5\% in most applications.

\section{Concluding remarks}

Chain-branching ignition in strained $\mathrm{H}_{2}$-air mixing layers has been addressed by analytical methods. It is seen that the chemistry description can be reduced by introducing a single radical-pool variable that incorporates the steady-state assumptions for $\mathrm{O}$ and $\mathrm{OH}$, avoiding the relatively large inaccuracies previously encountered with these assumptions. Extension of the proposed method to other chain-branching chemistries can be anticipated.

The results of the analysis enable calculations of ignition times through simple formulae such as (40) and (43) in terms of the first-mode eigenvalue $\lambda_{a}$, whose functional dependences on the flowfield conditions are exhibited in figures 3 and 4 . Following the approximate method proposed by Thévenin and Candel [4], these results are used for calculating the ignition distance in a shear layer, with predictions being in excellent agreement with the experimental observations. 
Weakly strained mixing layers are studied by addressing the limit of large Damköhler numbers, and a rigorous asymptotic description for the radical pool evolution is presented. With three terms in the asymptotic expansion for the growth rate, the range of validity of the results is seen to cover values of the Damköhler number $\Delta$ of the order of unity, corresponding to strain rates of the order of the critical ignition value. This unexpected extended range of validity enables the results of the asymptotics to be used, in general, in arriving at estimates of ignition times. The asymptotic description also indicates that, in weakly strained mixing layers, the leading-order radical growth rate $\lambda_{0} \simeq \kappa^{*}$ is independent of radical diffusion and corresponds to the maximum branching rate across the mixing layer. The associated ignition time is only weakly dependent on the strain rate imposed, in agreement with the results of previous numerical simulations of ignition in turbulent flows [17].

\section{Acknowledgments}

The work of JDM and ALS was supported by the Spanish DGESIC under project number PB98-0142-C04-02, while that of JSK was supported by the Korea Institute of Science and Technology under contract no 2E15530 and that of AL was supported by the Spanish CICYT under contract no PB94-0400 and by INTA under contract no INTA 4070-0036/1995.

\section{Appendix. The limit $\Delta \gg 1$}

In this limiting case, radical transport is apparently negligible in (29), and the resulting equation can be integrated to yield

$$
y=\left(\kappa_{1} / \kappa\right)\left(\mathrm{e}^{\kappa \tau}-1\right)
$$

an approximate solution that describes the initial development of the chain-branching explosion. The resulting profile shows a peak value that approaches $\eta=\eta^{*}$ for increasing values of $\tau$. Expanding the exponent in (A1) about $\eta^{*}$ according to $\kappa \tau=\kappa^{*} \tau+\left(\kappa^{\prime \prime} / 2\right)(\eta-$ $\left.\eta^{*}\right)^{2} \tau$, where $\kappa^{*}$ and $\kappa^{\prime \prime}$ denote the reduced branching rate and its second derivative evaluated at $\eta=\eta^{*}$, reveals that the thickness of the radical profile decreases with the reciprocal of the square root of time. Radical diffusion therefore becomes increasingly important, thereby leading to the failure of the simplified transportless description (A1) as $\tau$ increases.

In the solution that emerges, the branching process is confined to a thin layer located about $\eta=\eta^{*}$, whose characteristic thickness $\Delta^{-1 / 4}$ can be anticipated from (29) by imposing that the effect of radical diffusion and that of the variation of the branching rate must simultaneously enter in the reaction region for the solution to satisfy the imposed boundary conditions at $\eta= \pm \infty$. Combining this result with the previous estimate for the time dependence of the thickness of the radical profile (A1), one can conclude that (A1) remains approximately valid for $\tau \lesssim \Delta^{1 / 2}$, while radical diffusion must be taken into account to describe radical growth for $\tau \gtrsim \Delta^{1 / 2}$.

The analysis of this new regime employs a stretched coordinate

$$
\xi=\Delta^{1 / 4}\left(\eta-\eta^{*}\right)
$$

together with Taylor expansions of the form

$$
\begin{aligned}
& \kappa_{1}=\kappa_{1}^{*}+\Delta^{-1 / 4} \kappa_{1}^{\prime} \xi+\Delta^{-1 / 2} \kappa_{1}^{\prime \prime} \xi^{2} / 2+\cdots \\
& \kappa=\kappa^{*}+\Delta^{-1 / 2} \kappa^{\prime \prime} \xi^{2} / 2+\Delta^{-3 / 4} \kappa^{\prime \prime \prime} \xi^{3} / 6+\cdots
\end{aligned}
$$


and

$$
\frac{1}{L}=\frac{1}{L^{*}}+\Delta^{-1 / 4}\left(\frac{1}{L}\right)^{\prime} \xi+\Delta^{-1 / 2}\left(\frac{1}{L}\right)^{\prime \prime} \xi^{2} / 2+\cdots
$$

where $\kappa_{1}^{*}$ and $L^{*}$ denote the values of the functions $\kappa_{1}$ and $L$ at $\eta=\eta^{*}$ and the primes ' denote derivatives with respect to $\eta$ also evaluated at $\eta=\eta^{*}$.

Guided by the previous analysis of separation of variables, the development assumes a solution of the form

$$
y=\sum_{0}^{\infty} \alpha_{n}(\tau) \phi_{n}(\xi, \tau) T_{n}(\tau)
$$

where the coefficients $\alpha_{n}$ and the eigenfunctions $\phi_{n}$ are allowed to vary with time through the Damköhler-number variation according to the prescribed laws

$$
\alpha_{n}(\tau)=\pi_{n}^{0}+\Delta^{-1 / 4} \pi_{n}^{1}+\Delta^{-1 / 2} \pi_{n}^{2}+\cdots
$$

and

$$
\phi_{n}(\xi, \tau)=\psi_{n}^{0}(\xi)+\Delta^{-1 / 4} \psi_{n}^{1}(\xi)+\Delta^{-1 / 2} \psi_{n}^{2}(\xi)+\cdots .
$$

Introducing the above expressions into (29) with the initiation term expressed in the form

$$
\kappa_{1}=\sum_{0}^{\infty} \alpha_{n}(\tau) \phi_{n}(\xi, \tau)
$$

yields after separating variables

$$
\frac{1}{T_{n}}\left(\frac{\mathrm{d} T_{n}}{\mathrm{~d} \tau}-1\right)=\lambda_{n}(\tau)
$$

for the functions $T_{n}$, where an expansion of the form

$$
\lambda_{n}(\tau)=\kappa^{*}+\Delta^{-1 / 2} \Lambda_{n}^{0}+\Delta^{-3 / 4} \Lambda_{n}^{1}+\Delta^{-1} \Lambda_{n}^{2}+\cdots
$$

is assumed for the exponential growth rates. On the other hand, collecting terms in powers of $\Delta$ provides a set of differential equations for the functions $\psi_{n}^{i}(\xi)$, with $\Lambda_{n}^{i}$ entering as an eigenvalue in each of the resulting eigenvalue problems. For instance, collecting terms of order $\Delta^{-1 / 2}$ yields

$$
\frac{1}{L^{*}} \frac{\mathrm{d}^{2} \psi_{n}^{0}}{\mathrm{~d} \xi^{2}}-\left(\Lambda_{n}^{0}-\kappa^{\prime \prime} \xi^{2} / 2\right) \psi_{n}^{0}=0 \quad \psi_{n}^{0}( \pm \infty)=0
$$

for the leading-order functions $\psi_{n}^{0}$ and corresponding eigenvalues $\Lambda_{n}^{0}$. Solutions to (A12) exist only for a discrete set of values of $\Lambda_{n}^{0}$ given by

$$
\Lambda_{n}^{0}=-\sqrt{-2 \kappa^{\prime \prime} / L^{*}}\left(\frac{1}{2}+n\right)
$$

so that, for example,

$$
\Lambda_{0}^{0}=-\sqrt{-\kappa^{\prime \prime} /\left(2 L^{*}\right)}
$$

is the coefficient of the first correction in the expansion for $\lambda_{0}$. As expected, the corresponding eigenfunctions $\psi_{n}^{0}$ possess an increasing number of zeros, with the first-mode eigenfunction being

$$
\psi_{0}^{0}=\exp \left[-\left(-L^{*} \kappa^{\prime \prime} / 8\right)^{1 / 2} \xi^{2}\right]
$$


At leading order, equation (A9) becomes

$$
\kappa_{1}^{*}=\sum_{0}^{\infty} \pi_{n}^{0} \psi_{n}^{0}
$$

which can be solved for the coefficients $\pi_{n}^{0}=\kappa_{1}^{*} \int_{-\infty}^{\infty} \psi_{n}^{0} \mathrm{~d} \xi / \int_{-\infty}^{\infty}\left(\psi_{n}^{0}\right)^{2} \mathrm{~d} \xi$, yielding for example

$$
\pi_{0}^{0}=\sqrt{2} \kappa_{1}^{*}
$$

for the first mode.

Before carrying the solution to a higher order, it is worth investigating the influence of the higher modes on the solution. As can be observed in (A11), in the limit of large Damköhler numbers, all eigenvalues $\lambda_{n}$ converge asymptotically to the value $\kappa^{*}$, with differences between different $\lambda_{n}$ s being of order $\Delta^{-1 / 2}$. Therefore, the effect of the second and higher modes remains in the solution for values of $\tau$ smaller than or of order $\Delta^{1 / 2}$. Note that in this regime of $\tau \lesssim \Delta^{1 / 2}$ the terms of order $\Delta^{-1 / 2}$ and smaller in (A11) introduce only a small correction to the radical-pool variable $y$. Clearly, since $\tau \lesssim \Delta^{1 / 2}$ is precisely the range of validity of the transportless solution (A1), the sum of the infinite modes in (A6) yields in this regime $y=\left(\kappa_{1} / \kappa\right)\left(\mathrm{e}^{\kappa \tau}-1\right)$ in the first approximation.

On the other hand, for $\tau>\Delta^{1 / 2}$, the first mode becomes an accurate representation for the solution. Ignition is reached as $y$ increases to values of order $v$, which occurs for $\tau \sim \ln \left(v^{-1}\right)$. Therefore, the first mode dominates the solution, and determines in the first approximation the ignition time, in configurations with $\Delta \gg 1$ smaller than $\left[\ln \left(v^{-1}\right)\right]^{2}$, a very large quantity that takes the values $\left[\ln \left(v^{-1}\right)\right]^{2} \simeq 243$ and $\left[\ln \left(v^{-1}\right)\right]^{2}=101$ for $T_{\infty}=1200$ and $2000 \mathrm{~K}$, respectively. Therefore, under a wide range of strain conditions corresponding to $1 \ll \Delta \ll\left[\ln \left(v^{-1}\right)\right]^{2}$, the radical pool near ignition is accurately represented by the first mode $y=\alpha_{0}(\tau) \phi_{0}(\xi, \tau) T_{0}(\tau)$.

An arbitrary number of terms in the expansions for $\alpha_{0}, \phi_{0}$ and $\lambda_{0}$ can be computed by carrying the asymptotic method to a higher order. For instance, collecting terms of order $\Delta^{-3 / 4}$ yields the eigenvalue problem

$$
\frac{1}{L^{*}} \frac{\mathrm{d}^{2} \psi_{0}^{1}}{\mathrm{~d} \xi^{2}}-\left(\Lambda_{0}^{0}-\kappa^{\prime \prime} \xi^{2} / 2\right) \psi_{0}^{1}=\Lambda_{0}^{1} \psi_{0}^{0}-f_{1}(\xi) \quad \psi_{0}^{1}( \pm \infty)=0
$$

where

$$
f_{1}=\eta^{*} \frac{\mathrm{d} \psi_{0}^{0}}{\mathrm{~d} \xi}+\left(\frac{1}{L}\right)^{\prime} \frac{\mathrm{d}^{2}\left(\xi \psi_{0}^{0}\right)}{\mathrm{d} \xi^{2}}+\frac{1}{6} \kappa^{\prime \prime \prime} \xi^{3} \psi_{0}^{0}
$$

The solvability condition for (A18) requires that

$$
\Lambda_{0}^{1}=\frac{\int_{-\infty}^{+\infty} f_{1} \psi_{0}^{0} \mathrm{~d} \xi}{\int_{-\infty}^{+\infty}\left(\psi_{0}^{0}\right)^{2} \mathrm{~d} \xi}
$$

Since the function $f_{1}$ given in (A19) is an odd function of $\xi$ while $\psi_{0}^{0}$ is even, the integral in the numerator of the above expression vanishes, thereby giving

$$
\Lambda_{0}^{1}=0
$$

The simplified form of (A18) can then be solved to give

$$
\psi_{0}^{1}=-\psi_{0}^{0}\left\{L^{*} \xi\left[\frac{1}{6 L^{*}}+\left(\frac{1}{L}\right)^{\prime}+\frac{\eta^{*}}{2}\right]+\frac{\xi^{3}}{6}\left(-\frac{\kappa^{\prime \prime}\left(L^{*}\right)^{3}}{2}\right)^{1 / 2}\left[\frac{1}{3} \frac{\kappa^{\prime \prime \prime}}{\kappa^{\prime \prime} L^{*}}-\left(\frac{1}{L}\right)^{\prime}\right]\right\}
$$

